\title{
Comparación del cálculo de una estructura de pavimento flexible por elementos finitos y cálculo teórico.
}

\author{
Óscar Javier Reyes Ortiz. ${ }^{1}$, Javier Fernando Camacho Tauta ${ }^{2}$ \\ Grupo de investigación en geotecnia - Universidad Militar Nueva Granada
}

\author{
Freddy Reyes Lizcano. ${ }^{3}$ \\ Grupo CECATA - Universidad Javeriana
}

Fecha de recepción: 12 de abril de 2004

Fecha de aprobación : 21 de julio de 2004

\begin{abstract}
RESUMEN
El presente trabajo muestra una comparación entre los resultados obtenidos en deflexión, esfuerzo radial y esfuerzo vertical del cálculo de una estructura de pavimento flexible al ser analizada por el programa Praxis (Software de elementos finitos) y el programa DEPAV (software de cálculo analítico aproximado). La estructura de pavimento analizada consta de tres capas: la primera, una mezcla asfáltica, la segunda, una grava no tratada y la última, el suelo de soporte o subrasante. El estudio de la información se concentró en la capa de grava no tratada.
\end{abstract}

Con base en los resultados se puede concluir que para las propiedades de los materiales que constituyen la estructura de pavimento, la deflexión, el esfuerzo radial y el esfuerzo vertical calculados a partir del programa PLAXIS y el programa DEPAV son convergentes.

Palabras clave:

Pavimentos flexibles, PLAXIS, DEPAV, modelación numérica y elementos finitos.

\section{ABSTRACT}

This work presents a comparison between the obtained results the deflection, radial stress and vertical stress of the pavement structure being analyzed by the program PLAXIS (Finite element Software) and the DEPAV program (Analytical approximated calculus software). The pavement structure analyzed has got three layers: the first layer is an asphalt mixture, the second layer, a base untreated, and the last layer, the subgrade. The information analyzed was based on the base untreated layer.

According to the analysis, the concluded results were that the material proprieties that are part of the pavement structure, the deflection, radial stress and vertical stress calculated, in the PLAXIS and DEPAV programs, get to the same point.

\section{KEYWORDS}

Asphalt pavement, PLAXIS, DEPAV, finite element and numerical model.

\footnotetext{
${ }^{1}$ Ingeniero civil Universidad de Los Andes. Magíster Ingeniería Civil Universidad de Los Andes. Jefe del área de geotecnia.e-mail: oreyes@umng.edu.co

${ }^{2}$ Ingeniero civil Universidad Militar Nueva Granada. Magíster en Ingeniería Civil Universidad de Los

Andes. Docente - investigador. e-mail: jcamacho@umng.edu.co

${ }^{3}$ Ingeniero civil Universidad Javeriana. Docteur en Ingeniería Civil - Opción Pavimentos École Nationale Superieure de Mécanique y Laboratoire Centrale des Ponts et Chaussées Director de la especialización en geotecnia vial y pavimentos. e-mail: fredy.reyes@javeriana.edu.co
} 


\section{NOMENCLATURA}

$B_{i} \quad$ matriz cinemática que depende de la interpolación efectuada sobre el elemento $\mathrm{i}$

$D_{i} \quad$ matriz de comportamiento secante del material que depende de los esfuerzo $p$ y $q$

E Módulo de Young

$F \quad$ vector columna de las fuerzas modales

$K \quad$ matriz de rigidez de la estructura

Ka, Ga y $n$ Parámetros del modelo

$\mathrm{O}_{z} \quad$ origen de simetría axial

$P_{o} \quad$ estado inicial del masivo que es un estado de esfuerzos isotrópicos

Q carga total

d deflexión en $1 / 100 \mathrm{~mm}$

$h \quad$ espesor del material

$p \quad$ desviador isotrópico

$q \quad$ primer invariante de tensiones

$u \quad$ presión intersticial

$U$ desplazamiento horizontal

w desplazamiento vertical

$x \quad$ coeficiente que permite tener en cuenta el grado de saturación del suelo

$\varepsilon_{\mathrm{i}} \quad$ vector columna de las deformaciones del elemento $\mathrm{i}$

$\varepsilon_{\mathrm{q}} \quad$ deformación causada por el primer invariante de presiones

$\varepsilon_{\text {rr }} \quad$ deformación radial

$\varepsilon_{\mathrm{t}} \quad$ deformación cortante

$\varepsilon_{z} \quad$ deformación axial en la subrasante

$\varepsilon_{\mathrm{zz}} \quad$ deformación vertical

$\varepsilon_{\theta \theta} \quad$ deformación angular

$\sigma_{i} \quad$ vector columna de los esfuerzos del elemento i.

$\sigma_{\mathrm{rr}} \quad$ esfuerzo radial inicial

$\sigma_{\mathrm{rz}} \quad$ esfuerzo de cortante nulo

$\sigma_{\mathrm{zz}} \quad$ esfuerzo vertical inicial

$\sigma_{\theta \theta} \quad$ esfuerzo angular inicial

$v \quad$ relación de poisson

\section{INTRODUCCIÓN}

En la actualidad existen diferentes software que permiten realizar el diseño de estructuras de pavimento a partir de la teoría de elementos finitos, mecánica del medio continuo y métodos de análisis aproximados. En la mayoría de los análisis de diseño se supone que el material que constituye las capas de la estructura de pavimento trabaja en el rango elástico, es homogéneo, isotrópico y que las capas se adhieren perfectamente entre si, lo cual en la realidad es imposible lograr.

El propósito de este trabajo fue determinar las deflexiones, esfuerzos radiales y verticales de las capas de la estructura de pavimento flexible al emplear un programa de elementos finitos (PLAXIS 7.2), el cual puede analizar el comportamiento de la capa de grava no tratada como un material no lineal y compararlo con los resultados del programa DEPAV, el cual está fundamentado en las ecuaciones de la teoría de la elasticidad.

\section{MARCO CONCEPTUAL}


Los pavimentos son estructuras constituidas por un conjunto de capas superpuestas, las cuales se diseñan y construyen para soportar los esfuerzos y deformaciones que les generan las cargas del flujo vehicular. REF [1]

Para diseñar las estructuras de pavimento se emplean diferentes herramientas, entre las cuales se pueden enumerar los programas de elementos finitos, que están basados en la creación de un modelo bidimensional, el cual divide la región de estudio en triángulos de lados rectos, donde los vértices son los nodos y cada triangulo formado por tres nodos y tres lados, se llama elemento. REF [2] La solución de los problemas bidimensionales está resuelta como un esfuerzo plano y una deformación unitaria plana, la cual se basa en la Ley de Hooke (ecuación N. 1), con sus tres casos:

$\varepsilon_{i j}=\frac{1+v}{E} \sigma_{i j}-\frac{3 v}{E} \rho \delta_{i j} \quad$ Ecuación N. 1

Caso N. 1

El modelo es lineal, con módulo de elasticidad E y la relación de Poisson $v$ constantes, siendo estos los únicos parámetros del modelo.

\section{Caso N.2}

El modelo no es lineal y el módulo de elasticidad $\mathrm{E}$ y la relación de Poisson $v$ son función de los invariantes $p$ y $q$ que tiene el modelo de Boyce, el cual es:

$$
\boldsymbol{E}=\frac{\mathbf{9} \boldsymbol{K} \boldsymbol{G}}{\mathbf{3} \boldsymbol{K}+\boldsymbol{G}} \quad \text { Ecuación N. } 2
$$

$v=\frac{3 K-2 G}{6 K+2 G} \quad$ Ecuación N. 3

$$
\begin{aligned}
& K=K_{a}\left(\frac{p_{t}}{p_{a}}\right)^{1-n}\left[1-\frac{(1-n) K_{a}}{6 G_{a}}\left(\frac{q}{p_{t}}\right)^{2}\right]^{-1} \\
& G=G_{a}\left(\frac{p_{t}}{p_{a}}\right)^{1-n} \mathrm{y} \quad p_{t}=p_{0}+p, \quad \text { Ecuación N. } 4 \\
& \text { donde } \mathrm{Po}=\gamma_{\mathrm{i}} h_{i}
\end{aligned}
$$

\section{Caso N.3}

El modelo es no lineal, $v$ es constante y $E$ está determinado de la siguiente forma:

$$
E=E_{0}+\frac{E_{1}+P_{0}^{\prime}}{\rho \alpha},
$$

donde $P_{0}^{\prime}=P_{0}-\chi u$

Ecuación N. 5

y u es la presión intersticiial y $\chi$ es el grado de saturación del suelo.

El comportamiento experimental de gravas no tratadas se ha analizado con múltiples investigaciones de ensayos triaxiales, donde se pueden definir los invariantes $p$ y $q$ a partir de los esfuerzos principales. Para las estructuras de pavimento el estado inicial de esfuerzos y deformaciones es el debido al peso propio de los materiales y se determina como aparece en la ecuación N. 6 y los esfuerzos totales debidos a los invariantes $p$ y $q$ como está en las ecuaciones N. 7, 8, 9 y 10. REF [3] 
$\sigma_{r r}=\sigma_{\theta \theta}=\sigma_{z z}=P_{0}$

donde Ecuación N. 6

$\boldsymbol{P}_{0}=\sum_{i} \gamma_{i h_{i}}$

Ecuación N. 7

$P=\frac{1}{3}\left(\sigma_{r r}+\sigma_{\theta \theta}+\sigma_{z z}\right)$

Ecuación N. 8

$\varepsilon_{t}=\varepsilon_{r r}+\varepsilon_{\theta \theta}+\varepsilon_{z z}$

Ecuación N. 9

$q=\frac{1}{\sqrt{2}}\left[\left(\sigma_{r r}-\sigma_{\theta \theta}\right)^{2}+\left(\sigma_{\theta \theta}-\sigma_{z z}\right)^{2}+\left(\sigma_{z z}-\sigma_{r r}\right)^{2}+6 \cdot \varepsilon_{r z}^{2}\right]^{p .5}$

$\varepsilon q=\frac{\sqrt{2}}{3}\left[\left(\varepsilon_{r r}-\varepsilon_{\theta \theta}\right)^{2}+\left(\varepsilon_{\theta \theta}-\varepsilon_{z z}\right)^{2}+\left(\varepsilon_{z z}-\varepsilon_{r r}\right)^{2}+\mathbf{6} \cdot \varepsilon_{r z}{ }^{2}\right]^{0.5} \quad$ Ecuación N. 10

El comportamiento no lineal de las gravas no tratadas se soluciona con la matriz $[\mathrm{K}]$ como aparece en las ecuaciones N. 11 y 12; es importante resaltar que esta matriz es función de las deformaciones y esfuerzos de los nodos y que puede ser resuelto por el método secante para la iteración $n$, según se observa en la Figura N. 1. REF [4]
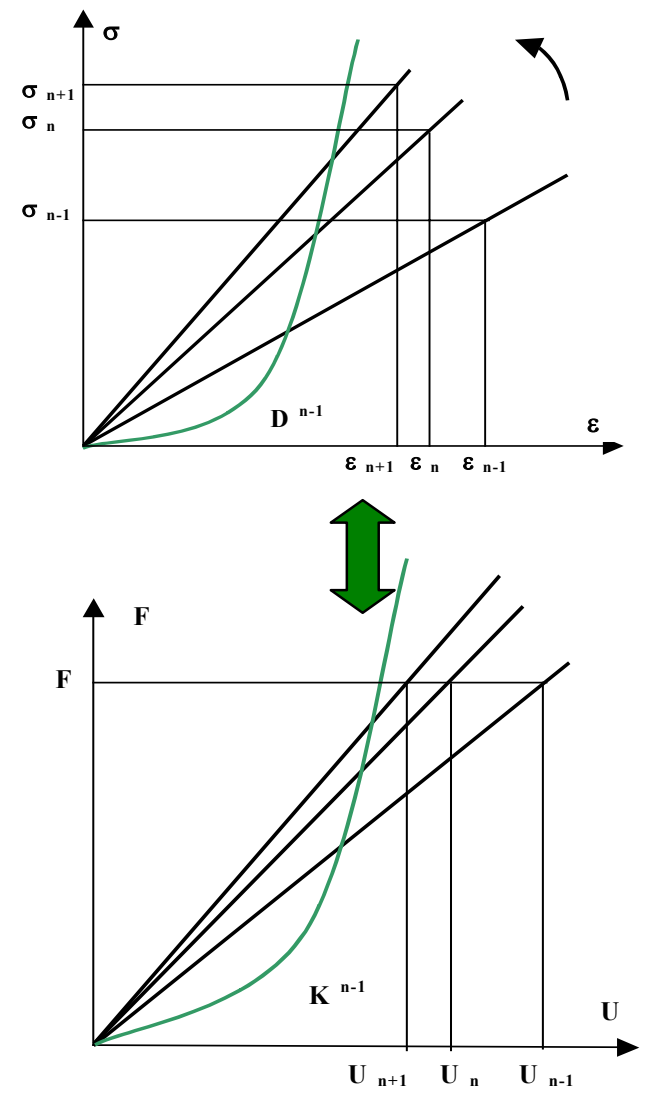

Figura 1. Esquematización del método secante

$\left\{U^{\mathrm{n}}\right\}=\left[K^{\mathrm{n}-1}\right]^{-1}\{F\}$

Ecuación N. 11 
$\left\{\sigma_{i}^{n}\right\}=\left[D_{i}^{n-1}\right]\left\{\varepsilon_{i}^{n}\right\}=\left[B_{i}\right]\left\{U^{n}\right\} \quad$ Ecuación N. 12

$\left[D_{i}{ }^{\circ}\right] \quad$ Se calcula a partir del campo de esfuerzos iniciales.

\section{METODOLOGÍA}

Para el desarrollo de la modelación de la estructura de pavimento flexible, se inició creando un sistema tripaca, donde la capa superior está constituida por una mezcla asfáltica, la intermedia por una grava no tratada y la inferior por una capa de suelo de soporte o subrasante.

Las cargas de los vehículos para el desarrollo de la investigación son semiejes estándar de $65 \mathrm{kN}$, que son esquematizadas como una presión de contacto de $0.662 \mathrm{Mpa}$. en un área circular con radio de 0.25 metros.

Las condiciones de frontera en el modelo analizado por elementos finitos fueron (Figura N. 2):

- En el borde inferior de la última capa, el desplazamiento vertical y el esfuerzo cortante son nulos. $\left(w=0\right.$ y $\left.\sigma_{\mathrm{rz}}=0\right)$

- En el borde lateral de las capas, el esfuerzo horizontal y el esfuerzo cortante son nulos $(U=0$ y $\left.\sigma_{\mathrm{rz}}=0\right)$.

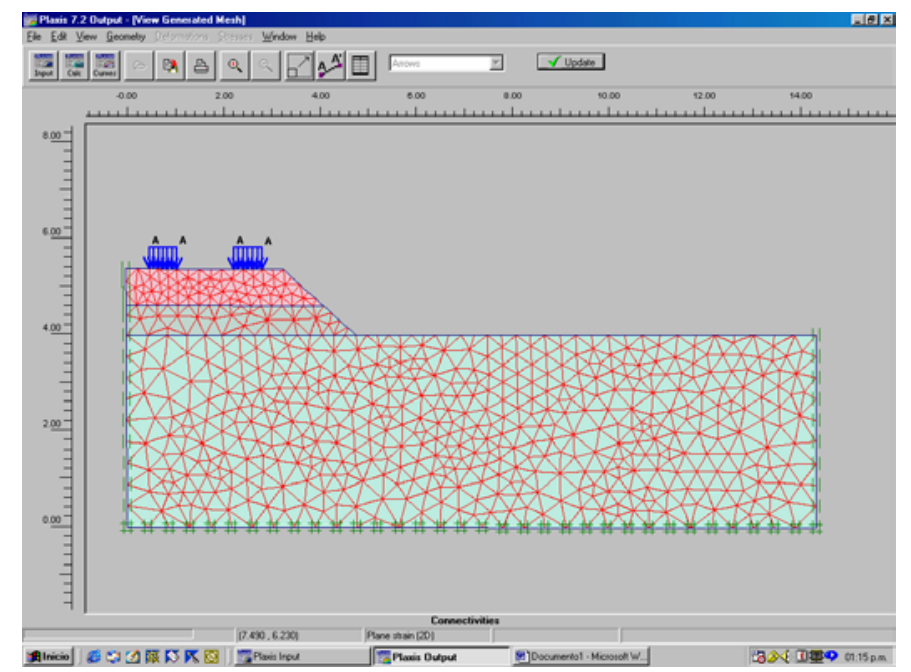

Figura N.2 (Modelo de la estructura de pavimentos en el programa PLAXIS) REF [5]

Las características de los materiales de la estructura de pavimento empleados son los que aparecen en la figura N.3 y donde los valores empleados para la matriz de rigidez de la estructura fueron $\mathrm{K}=2,10$ y 50 y el módulo de elasticidad de la subrasante es de $50 \mathrm{MPa}$. Es importante resaltar, que debido a que la capa de grava no tratada tiene un espesor de 40 centímetros, para el análisis del modelo se dividió en dos capas de 20 centímetros cada una y se determinó la resistencia de la parte superior de la capa en función de las propiedades elásticas de la parte inferior y que el tercer estrato (subrasante), para el programa PLAXIS, se tomó con un espesor de 5 metros, esto con el objeto de omitir los efectos de borde dentro del modelo. Para el caso del programa DEPAV, este estrato es considerado como infinito. 


\begin{tabular}{|lll|}
\hline Espesor $0.1 \mathrm{~m}$ & $\mathrm{v}=0.4$ & $\mathrm{E}_{1}=5000 \mathrm{MPa}$. \\
\hline Espesor $0.2 \mathrm{~m}$ & $\mathrm{v}=0.25$ & $\mathrm{E}_{2}=\mathrm{K} \mathrm{E}_{3}$ \\
\hline Espesor $0.2 \mathrm{~m}$ & $\mathrm{v}=0.25$ & $\mathrm{E}_{3}=\mathrm{K} \mathrm{E}_{4}$ \\
\hline & \\
& \\
Espesor $5 \mathrm{~m}$ & $\mathrm{v}=0.45$ & \\
& \\
& \\
& \\
\end{tabular}

Figura N. 3 Características de las capas de la estructura de pavimentos.

\section{ANÁLISIS DE DATOS}

En la tabla N.1 se observan los valores calculados de los esfuerzos verticales $\left(\sigma_{z z}\right)$, esfuerzos radiales $\left(\sigma_{\mathrm{rr}}\right)$ y deflexiones $(d)$ por los programas PLAXIS y DRPAV en la capa de grava no tratada y con los tres valores de la matriz de rigidez. (Caso $1, \mathrm{~K}=2$, caso $2, \mathrm{~K}=10$ y caso $3, \mathrm{~K}=50$ ).

\begin{tabular}{|c|c|c|c|c|}
\hline Caso & Programa & $\boldsymbol{d}(\mathbf{1 / 1 0 0} \mathbf{m m})$ & $\sigma_{\mathbf{r r}} \mathbf{( K P a )}$ & $\sigma_{\mathbf{z z}}(\mathbf{K P a})$ \\
\hline 1 & PLAXIS & 1.69 & -77 & 60 \\
& DEPAV & 1.67 & -57 & 59.5 \\
\hline 2 & PLAXIS & 0.43 & -941 & 22.1 \\
& DEPAV & 0.46 & -940 & 21.1 \\
\hline \multirow{2}{*}{3} & PLAXIS & 0.22 & -2660 & 7.2 \\
& DePAV & 0.15 & -2800 & 5.6 \\
\hline
\end{tabular}

Tabla N. 1 Resultados de los esfuerzos y deflexiones de la capa de grava no tratada.

Al comparar porcentualmente los resultados obtenidos de los dos programas y teniendo como patrón el software de PLAXIS, se pudo determinar que para el caso N.1, la deflexión se reducía en $1.2 \%$, el esfuerzo vertical en $0.8 \%$ y el esfuerzo radial en $26 \%$. Para el caso N.2, se incremento en $7 \%$ la deflexión, se reduce en $0.1 \%$ el esfuerzo radial y en $4.5 \%$ el esfuerzo vertical. Por último, el caso 3 , la deflexión se redujo en $32 \%$, el esfuerzo radial se incrementó en un $5.3 \%$ y se redujo en $22.3 \%$ el esfuerzo vertical.

\section{CONCLUSIONES}

Una vez analizada la información de los resultados obtenidos en los programas PLAXIS y DEPAV, se pudo concluir que el comportamiento lineal y no lineal de la capa de grava no tratada fue:

- Para los valores de rigidez de 2 y 10 , el comportamiento de las deflexiones converge, existiendo una desviación del orden de $1.2 \%$. 
- En el caso de la rigidez de $\mathrm{K}=50$, el comportamiento de la deflexión, el esfuerzo radial y el esfuerzo vertical, conducen a errores aproximadamente de $32 \%, 5 \%$ y $22 \%$ respectivamente, llevando a malas apreciaciones en el diseño de estructuras al comparar los resultados de los dos programas.

- Con respecto al esfuerzo radial para el caso N. 1, la desviación encontrada fue de $26 \%$.

\section{BIBLIOGRAFÍA}

[1] IDU-Uniandes. 2002. Reglamento Técnico Vial - 2002, Ed. Legis.

[2] chandrupatla, t.1999 Introducción al estudio del elemento finito en ingeniería. Editorial Prentice Hall. Segunda edición.

[3] Boyce h.r, 1980, "A non-linear model for the elastic behaviour of granular materials under repeated loading”, Inter. Symposioum o Soils under Ciclic loading Swansea pp. 285-294

[4] De boissoudy a. b, 1987, "La prise en compte des graves non traitées dans le dimensionement, lévolution des méthodes de calcul", LCPC.

[5] Plaxis, Manual versión 7.2

[6] Reyes lizcano, f. 2003. Diseño Racional de Pavimentos. Editorial Escuela Colombiana de Ingeniería.

[7] Brown s.f barsdale r.d, 1987), "Pavement design and materials", proc $6^{\mathrm{TH}}$ Inter. Conference on the Structural Design of Asfalt Pavement, Ann Arbor, Vol 1, pp. 118-148.

[8] Elhannani m, 1991, "Modelation et simulation numérique des chaussés souples", These de doctorat université de Nantes.

[9]

Humbert p. cesar, 1989, "Calcul aux éléments finis", Bull liaison Labo P et Ch, LCPC.

[10] Martínez j., 1990, "Dimensionnement rationnel des structures de chaussés souple et inverses". These de doctorat, université de Montpellier.

[11]

Paute j. I., jouve p., martínez, "Modéle de calcul pour le dimensionnement des chaussés souple, Bull", liaison Labo P et Ch, 196 juil-aout, 1998. 